\title{
High Efficient Numerical Methods for Viscous and Nonviscous Wave Problems
}

\author{
Xiujie Lv, ${ }^{1}$ Jinggang Qin, ${ }^{2}$ and Tongke Wang ${ }^{3}$ \\ ${ }^{1}$ Center for Combinatorics, Nankai University, Tianjin 300071, China \\ ${ }^{2}$ Institute of Plasma Physics, Chinese Academy of Sciences, Hefei, Anhui Province 230031, China \\ ${ }^{3}$ School of Mathematical Sciences, Tianjin Normal University, Tianjin 300387, China \\ Correspondence should be addressed to Xiujie Lv; lxiuj@163.com
}

Received 9 October 2012; Revised 31 March 2013; Accepted 22 April 2013

Academic Editor: Jae-Wook Kim

Copyright (C) 2013 Xiujie Lv et al. This is an open access article distributed under the Creative Commons Attribution License, which permits unrestricted use, distribution, and reproduction in any medium, provided the original work is properly cited.

\begin{abstract}
This paper is concerned with accurate and efficient numerical methods for solving viscous and nonviscous wave problems. The paper first introduces a new second-order PR-ADI like scheme. For an efficient simulation, the scheme is also extended to a highorder compact PRADI like method. Both of them have the advantages of unconditional stability, less impact of the perturbing terms on the accuracy, and being convenient to compute the boundary values of the intermediates. Besides this, the compact scheme has high-order accuracy and costs less in computational time. Numerical results are presented to show the accuracy and efficiency of the new algorithms.
\end{abstract}

\section{Introduction}

In this paper, we consider the following differential problem with initial and boundary conditions on domain $\Omega=[0,1]^{2}$ :

$$
\begin{array}{r}
\gamma_{1} \frac{\partial u}{\partial t}+\gamma_{2} \frac{\partial^{2} u}{\partial t^{2}}-a \Delta u-q \frac{\partial}{\partial t} \Delta u=S(x, y, t), \\
(x, y) \in \Omega, t \in(0, T], \\
u(x, y, 0)=u_{0}(x, y), \quad \frac{\partial u}{\partial t}(x, y, 0)=\psi(x, y), \\
(x, y) \in \Omega, \\
u(x, y, t)=\varphi(x, y, t), \quad(x, y) \in \partial \Omega, t \in(0, T],
\end{array}
$$

where $\gamma_{1}, \gamma_{2}, a$, and $q$ are nonnegative constants, and $a \gamma_{2}>$ $\gamma_{1} q, S$ denotes the source, and $u_{0}, \psi$, and $\varphi$ are given functions, and $\partial \Omega$ is the boundary of the domain $\Omega$.

Equations (1)-(3) govern various physical phenomena by choosing the coefficients. When $\gamma_{2}=0$ and $q=0$, the equation represents standard heat transfer, and when $\gamma_{1}=0$ and $q=0$, the equation represents standard wave equations. Since there are many efficient algorithms known for them, we exclude their consideration.
Some conventional numerical approaches for solving wave equations introduce auxiliary variables to rewrite the equations as first-order hyperbolic systems [1-3]; however, these approaches introduce new unknowns which results in an increase in the number of variables in the discrete problems. Thus, there are advantages in keeping the formulation (1)-(3) involving the second time-derivative and a scalar unknown. It has been the case that it is hard to construct methods combining good stability with high accuracy with this formulation [4].

This paper is concerned with numerical solutions to viscous and nonviscous nonhomogeneous wave problems. The wave equation is often solved by explicit time-stepping schemes, which require to choose a time step size sufficiently small to satisfy the stability condition and to reduce numerical dispersion as well. As is well known, the alternating direction implicit (ADI) schemes [3-14] are unconditionally stable and only need to solve a sequence of tridiagonal linear systems. In 1955, Peaceman and Rachford Jr. [15] presented a method to solve two-dimensional parabolic equations. When constructing the difference scheme, they first evaluated the derivative with respect to $x$ implicitly, the derivative with respect to $y$ explicitly and used a time step of $\Delta t / 2$ to get a scheme, then took another half step with explicit in 
$x$ and implicit in $y$ to get another scheme. The final twostep scheme, called Peaceman and Rachford (PR) scheme, is unconditionally stable and second-order accurate. Alternative conventional approaches for solving wave equations introduce an auxiliary variable to rewrite the equation as a first-order hyperbolic system. With these approaches one introduces new unknowns, which result in an increase in the number of variables in the discrete problems. Thus, there are good reasons to try to keep the formulation involving the second time-derivative and a scalar unknown. Lim et al. [11] introduce a stable three-level locally one-dimensional (LOD) method for solving (1)-(3), which is second-order in space.

In the context of high order finite difference methods, compact schemes feature high-order accuracy and small stencils. Recently, there has been a renewed interest in the development and the application of compact finite difference methods for the numerical solution of the differential equations $[8-10,12,14]$. To obtain satisfactory higher order numerical results with reasonable computational cost, there have been attempts to develop higher order compact ADI methods.

In this paper, we propose a new set of ADI methods for viscous and nonviscous wave problems. In Section 3, we obtain a new PR ADI-like scheme with truncation error $O\left(\Delta t^{2}+h^{2}\right)$ and analyze the error estimate by discrete energy method. In Section 4, we further extend the method to compact PR ADI-like scheme with truncation error $\mathrm{O}\left(\Delta t^{2}+\right.$ $h^{4}$ ). In Section 5, we provide two numerical examples to illustrate the effectiveness of the scheme. We also compare the two methods with some other schemes and the results show that they have much higher efficiency.

\section{Notation}

The domain $\Omega$ is divided into a mesh by points $x_{i}=i h, y_{j}=$ $j h(i, j=0, \ldots, N)$, denoted by $\Omega_{h}$, where $h=1 / N$ is the spatial mesh size in both $x$ and $y$ directions. Let $t^{n}=$ $n \Delta t, t^{n+1 / 2}=(n+1 / 2) \Delta t$, with $\Delta t$ being the time increment. Denote the values of $u\left(x_{i}, y_{j}, t^{n}\right)$ and $S\left(x_{i}, y_{j}, t^{n+1 / 2}\right)$ by $u_{i, j}^{n}$ and $S_{i, j}^{n+1 / 2}$, respectively. Denote also the difference solutions to $u_{i, j}^{n}$ and $v_{i, j}^{n}$ by $U_{i, j}^{n}$ and $V_{i, j}^{n}$, respectively.

Assume that $V_{h}=\left\{w \mid w=\left\{w_{i, j}\right\} \in \Omega_{h}\right.$, and $\left.\left.w\right|_{\partial \Omega}=0\right\}$. For $w=\left\{w_{i, j}\right\} \in V_{h}$ and $v=\left\{v_{i, j}\right\} \in V_{h}$, denote by

$$
\begin{gathered}
\delta_{t} v_{i, j}^{n}=\frac{v_{i, j}^{n+1}-v_{i, j}^{n}}{\Delta t}, \quad \delta_{x} v_{i, j}^{n}=\frac{v_{i+1, j}^{n}-v_{i, j}^{n}}{h}, \\
\delta_{y} v_{i, j}^{n}=\frac{v_{i, j+1}^{n}-v_{i, j}^{n}}{h}, \\
\delta_{x}^{2} v_{i, j}^{n}=\frac{v_{i+1, j}^{n}-2 v_{i, j}^{n}+v_{i-1, j}^{n}}{h^{2}}, \\
\delta_{y}^{2} v_{i, j}^{n}=\frac{v_{i, j+1}^{n}-2 v_{i, j}^{n}+v_{i, j-1}^{n}}{h^{2}} .
\end{gathered}
$$

For $\forall v, w \in V_{h}$, define their discrete inner product and norms as follows:

$$
\begin{gathered}
(v, w)=\sum_{i, j=1}^{N-1} v_{i, j} w_{i, j} h^{2},\|v\|=\sqrt{(v, v),} \\
\left\|\delta_{x} v\right\|=\sqrt{\sum_{i=0}^{N-1} \sum_{j=1}^{N-1}\left(\delta_{x} v_{i, j}\right)^{2} h^{2}} \\
\left\|\delta_{y} v\right\|=\sqrt{\sum_{i=1}^{N-1} \sum_{j=0}^{N-1}\left(\delta_{y} v_{i, j}\right)^{2} h^{2}} \\
\left\|\delta_{x} \delta_{y} v\right\|=\sqrt{\sum_{i=0}^{N-1} \sum_{j=0}^{N-1}\left(\delta_{x} \delta_{y} v_{i, j}\right)^{2} h^{2}} \\
|v|_{H^{1}}=\sqrt{\left\|\delta_{x} v\right\|^{2}+\left\|\delta_{y} v\right\|^{2}} .
\end{gathered}
$$

\section{The New Second-Order ADI Scheme for Two-Dimensional Problems}

In this section, we derive a new second-order ADI method for the numerical solution of the differential problems (1)-(3).

3.1. Construction of the New ADI Scheme. Introducing $v=$ $\gamma_{1} u+\gamma_{2}(\partial u / \partial t)$, we can rewrite (1) as follows:

$$
\begin{gathered}
\frac{\partial v}{\partial t}-\alpha \triangle v-\beta \triangle u=S, \quad(x, y) \in \Omega, t \in(0, T], \\
v=\gamma_{1} u+\gamma_{2} \frac{\partial u}{\partial t}, \\
u(x, y, 0)=u_{0}(x, y), \\
v(x, y, 0)=\gamma_{1} u_{0}(x, y)+\gamma_{2} \psi(x, y), \\
u(x, y, t)=\varphi(x, y, t), \quad(x, y) \in \Omega, \\
v(x, y, t)=\gamma_{1} \varphi(x, y, t)+\gamma_{2} \varphi_{t}(x, y, t), \\
(x, y) \in \partial \Omega, t \in(0, T],
\end{gathered}
$$

where $\alpha=q / \gamma_{2}, \beta=a-\left(\gamma_{1} q / \gamma_{2}\right)$.

Applying Crank-Nicolson implicit discretization to (6), we have

$$
\begin{gathered}
\frac{V_{i, j}^{n+1}-V_{i, j}^{n}}{\Delta t}-\alpha\left(\delta_{x}^{2}+\delta_{y}^{2}\right) \frac{V_{i, j}^{n+1}+V_{i, j}^{n}}{2} \\
-\beta\left(\delta_{x}^{2}+\delta_{y}^{2}\right) \frac{U_{i, j}^{n+1}+U_{i, j}^{n}}{2}=S_{i, j}^{n+1 / 2}, \\
\frac{V_{i, j}^{n+1}+V_{i, j}^{n}}{2}=\gamma_{1} \frac{U_{i, j}^{n+1}+U_{i, j}^{n}}{2}+\gamma_{2} \frac{U_{i, j}^{n+1}-U_{i, j}^{n}}{\Delta t} .
\end{gathered}
$$


From (10), we obtain

$$
\begin{aligned}
V_{i, j}^{n+1}- & \frac{\Delta t}{2} \alpha\left(\delta_{x}^{2}+\delta_{y}^{2}\right) V_{i, j}^{n+1}-\frac{\Delta t}{2} \beta\left(\delta_{x}^{2}+\delta_{y}^{2}\right) U_{i, j}^{n+1} \\
= & V_{i, j}^{n}+\frac{\Delta t}{2} \alpha\left(\delta_{x}^{2}+\delta_{y}^{2}\right) V_{i, j}^{n}+\frac{\Delta t}{2} \beta\left(\delta_{x}^{2}+\delta_{y}^{2}\right) U_{i, j}^{n} \\
& +\Delta t S_{i, j}^{n+1 / 2}, \\
U_{i, j}^{n+1} & =\frac{\Delta t}{\gamma_{1} \Delta t+2 \gamma_{2}}\left(V_{i, j}^{n+1}+V_{i, j}^{n}\right)-\frac{\gamma_{1} \Delta t-2 \gamma_{2}}{\gamma_{1} \Delta t+2 \gamma_{2}} U_{i, j}^{n} .
\end{aligned}
$$

Let

$$
\begin{gathered}
\lambda_{1}=\frac{\Delta t}{\gamma_{1} \Delta t+2 \gamma_{2}}, \quad \lambda_{2}=\frac{\gamma_{1} \Delta t-2 \gamma_{2}}{\gamma_{1} \Delta t+2 \gamma_{2}}, \\
c=\alpha+\beta \lambda_{1}, \quad \gamma=\frac{1}{2} \beta\left(1-\lambda_{2}\right),
\end{gathered}
$$

then (11) can be reorganized as

$$
\begin{aligned}
V_{i, j}^{n+1}- & \frac{c \Delta t}{2}\left(\delta_{x}^{2}+\delta_{y}^{2}\right) V_{i, j}^{n+1} \\
= & V_{i, j}^{n}+\frac{c \Delta t}{2}\left(\delta_{x}^{2}+\delta_{y}^{2}\right) V_{i, j}^{n}+\gamma \Delta t\left(\delta_{x}^{2}+\delta_{y}^{2}\right) U_{i, j}^{n} \\
& +\Delta t S_{i, j}^{n+1 / 2}
\end{aligned}
$$

Adding perturbing term $\left(c^{2} \Delta t^{2} / 4\right) \delta_{x}^{2} \delta_{y}^{2}\left(V_{i, j}^{n+1}-V_{i, j}^{n}\right)$ to the left hand side of (14), we factor (14) as

$$
\begin{aligned}
\left(1-\frac{c \Delta t}{2} \delta_{x}^{2}\right)\left(1-\frac{c \Delta t}{2} \delta_{y}^{2}\right) V_{i, j}^{n+1} \\
=\left(1+\frac{c \Delta t}{2} \delta_{x}^{2}\right)\left(1+\frac{c \Delta t}{2} \delta_{y}^{2}\right) V_{i, j}^{n} \\
\quad+\gamma \Delta t\left(\delta_{x}^{2}+\delta_{y}^{2}\right) U_{i, j}^{n}+\Delta t S_{i, j}^{n+1 / 2} .
\end{aligned}
$$

Introducing the intermediate variable $V_{i, j}^{n+1 / 2}$, we obtain the PR ADI-like scheme as follows:

$$
\begin{aligned}
\left(1-\frac{c \Delta t}{2} \delta_{x}^{2}\right) V_{i, j}^{n+1 / 2}= & \left(1+\frac{c \Delta t}{2} \delta_{y}^{2}\right) V_{i, j}^{n} \\
& +\gamma\left(\frac{\Delta t}{2} \delta_{y}^{2}+\frac{1}{c}\right) U_{i, j}^{n}+\frac{\Delta t}{2} S_{i, j}^{n+1 / 2}, \\
\left(1-\frac{c \Delta t}{2} \delta_{y}^{2}\right) V_{i, j}^{n+1}= & \left(1+\frac{c \Delta t}{2} \delta_{x}^{2}\right) V_{i, j}^{n+1 / 2} \\
& +\gamma\left(\frac{\Delta t}{2} \delta_{y}^{2}-\frac{1}{c}\right) U_{i, j}^{n}+\frac{\Delta t}{2} S_{i, j}^{n+1 / 2} \\
U_{i, j}^{n+1}=\lambda_{1}( & \left.V_{i, j}^{n+1}+V_{i, j}^{n}\right)-\lambda_{2} U_{i, j}^{n} .
\end{aligned}
$$

From (16), (17), we get the boundary equation as follows:

$$
V_{i, j}^{n+1 / 2}=\frac{V_{i, j}^{n+1}+V_{i, j}^{n}}{2}+\frac{\gamma}{c} U_{i, j}^{n}-\frac{c \Delta t}{4} \delta_{y}^{2}\left(V_{i, j}^{n+1}-V_{i, j}^{n}\right) .
$$

But usually we compute the boundary values of the intermediate variable using the following simple equality:

$$
V_{i, j}^{n+1 / 2}=\frac{V_{i, j}^{n+1}+V_{i, j}^{n}}{2}+\frac{\gamma}{c} U_{i, j}^{n}
$$

where $i=0, N, j=0, \ldots, N-1$.

We see that schemes (16)-(20) can solve differential problems (1)-(3). In addition, following the idea of Douglas $([2,3])$, a Douglas-like scheme is as follows:

$$
\begin{aligned}
& \left(1-\frac{c \Delta t}{2} \delta_{x}^{2}\right)\left(V_{i, j}^{n+1 / 2}-V_{i, j}^{n}\right) \\
& \quad=c \Delta t\left(\delta_{x}^{2}+\delta_{y}^{2}\right) V_{i, j}^{n}+\gamma \Delta t\left(\delta_{x}^{2}+\delta_{y}^{2}\right) U_{i, j}^{n}+\Delta t S_{i, j}^{n+1 / 2},
\end{aligned}
$$

$$
\left(1-\frac{c \Delta t}{2} \delta_{y}^{2}\right)\left(V_{i, j}^{n+1}-V_{i, j}^{n}\right)=V_{i, j}^{n+1 / 2}-V_{i, j}^{n}
$$

The intermediate value of $V_{i, j}^{n+1 / 2}$ on the boundary is easy to be obtained from (22). PR ADI-like scheme (16)-(18) and Douglas-like scheme (21)-(22) are all derived from equation (15), but PR ADI-like scheme has more efficiency, which will be found in numerical experiment.

3.2. Error Estimate. We have derived a new kind of ADI scheme in Section 3.1. We further analyze the error of the scheme starting from (15). Expanding (15), we get

$$
\begin{aligned}
& \frac{V_{i, j}^{n+1}-V_{i, j}^{n}}{\Delta t}-\alpha\left(\delta_{x}^{2}+\delta_{y}^{2}\right) \frac{V_{i, j}^{n+1}+V_{i, j}^{n}}{2} \\
& \quad-\beta\left(\delta_{x}^{2}+\delta_{y}^{2}\right) \frac{U_{i, j}^{n+1}+U_{i, j}^{n}}{2}+\frac{c^{2} \Delta t^{2}}{4} \delta_{x}^{2} \delta_{y}^{2} \frac{V_{i, j}^{n+1}-V_{i, j}^{n}}{\Delta t} \\
& \quad=S_{i, j}^{n+1 / 2} .
\end{aligned}
$$

Discretizing (6) as above and letting $\xi=u-U, \eta=v-V$, we obtain the error equations as follows:

$$
\begin{aligned}
& \frac{\eta_{i, j}^{n+1}-\eta_{i, j}^{n}}{\Delta t}-\alpha\left(\delta_{x}^{2}+\delta_{y}^{2}\right) \frac{\eta_{i, j}^{n+1}+\eta_{i, j}^{n}}{2}-\beta\left(\delta_{x}^{2}+\delta_{y}^{2}\right) \frac{\xi_{i, j}^{n+1}+\xi_{i, j}^{n}}{2} \\
& \quad+\frac{c^{2} \Delta t^{2}}{4} \delta_{x}^{2} \delta_{y}^{2} \frac{\eta_{i, j}^{n+1}-\eta_{i, j}^{n}}{\Delta t}=R_{i, j}^{n+1 / 2},
\end{aligned}
$$

$$
\frac{\eta_{i, j}^{n+1}+\eta_{i, j}^{n}}{2}=\gamma_{1} \frac{\xi_{i, j}^{n+1}+\xi_{i, j}^{n}}{2}+\gamma_{2} \frac{\xi_{i, j}^{n+1}-\xi_{i, j}^{n}}{\Delta t}+\widetilde{R}_{i, j}^{n+1 / 2}
$$


where the truncation errors

$$
\begin{aligned}
& R_{i, j}^{n+1 / 2} \\
& =\Delta t^{2}\left(\frac{1}{24} \frac{\partial^{3} v}{\partial t^{3}}-\frac{\alpha}{8}\left(\frac{\partial^{4} v}{\partial t^{2} \partial x^{2}}+\frac{\partial^{4} v}{\partial t^{2} \partial y^{2}}\right)\right. \\
& -\frac{\beta}{8}\left(\frac{\partial^{4} u}{\partial t^{2} \partial x^{2}}+\frac{\partial^{4} u}{\partial t^{2} \partial y^{2}}\right) \\
& \left.+\frac{c^{2} \Delta t^{2}}{4} \frac{\partial^{5} v}{\partial t \partial x^{2} \partial y^{2}}\right)\left(x_{i}, y_{j}, t^{n+1 / 2}\right) \\
& -\frac{\alpha h^{2}}{12}\left(\frac{\partial^{4} v}{\partial x^{4}}+\frac{\partial^{4} v}{\partial y^{4}}\right)\left(x_{i}, y_{j}, t^{n+1 / 2}\right) \\
& -\frac{\beta h^{2}}{12}\left(\frac{\partial^{4} u}{\partial x^{4}}+\frac{\partial^{4} u}{\partial y^{4}}\right)\left(x_{i}, y_{j}, t^{n+1 / 2}\right) \\
& +O\left(\Delta t^{4}+\Delta t^{2} h^{2}+h^{4}\right), \\
& \widetilde{R}_{i, j}^{n+1 / 2}=\Delta t^{2}\left(\frac{1}{8} \frac{\partial^{2} v}{\partial t^{2}}-\frac{\gamma_{1}}{8} \frac{\partial^{2} u}{\partial t^{2}}-\frac{\gamma_{2}}{24} \frac{\partial^{3} u}{\partial t^{3}}\right)\left(x_{i}, y_{j}, t^{n+1 / 2}\right) \\
& +O\left(\Delta t^{4}\right) \text {. }
\end{aligned}
$$

Hence there exist positive constants $C_{1}, C_{2}$ such that

$$
\left|R_{i, j}^{n+1 / 2}\right| \leq C_{1}\left(\Delta t^{2}+h^{2}\right), \quad\left|\widetilde{R}_{i, j}^{n+1 / 2}\right| \leq C_{2} \Delta t^{2}
$$

Lemma 1 (see [1]). For $w \in V_{h}$, the following equalities hold:

$$
\begin{aligned}
-\sum_{i=1}^{N-1} \sum_{j=1}^{N-1} w_{i, j}\left(\delta_{x}^{2} w_{i, j}\right) h^{2} & =\sum_{i=0}^{N-1} \sum_{j=1}^{N-1}\left(\delta_{x} w_{i, j}\right)^{2} h^{2}=\left\|\delta_{x} w\right\|^{2} \\
-\sum_{i=1}^{N-1} \sum_{j=1}^{N-1} w_{i, j}\left(\delta_{y}^{2} w_{i, j}\right) h^{2} & =\sum_{i=1}^{N-1} \sum_{j=0}^{N-1}\left(\delta_{y} w_{i, j}\right)^{2} h^{2}=\left\|\delta_{y} w\right\|^{2} .
\end{aligned}
$$

From Lemma 1 and Cauchy-Schwarz inequality, multiplying $\left(\left(\eta_{i, j}^{n+1}+\eta_{i, j}^{n}\right) / 2\right) h^{2}$ to both sides of (24), computing the inner product, it is easy to follow that

$$
\begin{aligned}
& I_{1}=\left(\frac{\eta^{n+1}-\eta^{n}}{\Delta t}, \frac{\eta^{n+1}+\eta^{n}}{2}\right)=\frac{1}{2 \Delta t}\left(\left\|\eta^{n+1}\right\|^{2}-\left\|\eta^{n}\right\|^{2}\right), \\
& I_{2_{1}}=-\left(\alpha \delta_{x}^{2} \frac{\eta^{n+1}+\eta^{n}}{2}, \frac{\eta^{n+1}+\eta^{n}}{2}\right)=\alpha\left\|\delta_{x} \frac{\eta^{n+1}+\eta^{n}}{2}\right\|^{2}, \\
& I_{2_{2}}=-\left(\alpha \delta_{y}^{2} \frac{\eta^{n+1}+\eta^{n}}{2}, \frac{\eta^{n+1}+\eta^{n}}{2}\right)=\alpha\left\|\delta_{y} \frac{\eta^{n+1}+\eta^{n}}{2}\right\|^{2},
\end{aligned}
$$

$$
\begin{aligned}
& I_{3_{1}}=-\left(\beta \delta_{x}^{2} \frac{\xi^{n+1}+\xi^{n}}{2}, \frac{\eta^{n+1}+\eta^{n}}{2}\right) \\
& =-\beta\left(\delta_{x}^{2} \frac{\xi^{n+1}+\xi^{n}}{2}, \frac{\eta^{n+1}+\eta^{n}}{2}\right) \\
& =-\beta\left(\delta_{x}^{2} \frac{\xi^{n+1}+\xi^{n}}{2},\right. \\
& \left.\gamma_{1} \frac{\xi^{n+1}+\xi^{n}}{2}+\gamma_{2} \frac{\xi^{n+1}-\xi^{n}}{\Delta t}+\widetilde{R}^{n+1 / 2}\right) \\
& =\beta \gamma_{1}\left\|\delta_{x} \frac{\xi^{n+1}+\xi^{n}}{2}\right\|^{2}+\frac{\beta \gamma_{2}}{2 \Delta t}\left(\left\|\delta_{x} \xi^{n+1}\right\|^{2}-\left\|\delta_{x} \xi^{n}\right\|^{2}\right) \\
& -\left[-\beta\left(\delta_{x} \frac{\xi^{n+1}+\xi^{n}}{2}, \delta_{x} \widetilde{R}^{n+1 / 2}\right)\right] \\
& \geq \frac{\beta \gamma_{2}}{2 \Delta t}\left(\left\|\delta_{x} \xi^{n+1}\right\|^{2}-\left\|\delta_{x} \xi^{n}\right\|^{2}\right) \\
& -\left\|\beta \delta_{x} \frac{\xi^{n+1}+\xi^{n}}{2}\right\|\left\|\delta_{x} \widetilde{R}^{n+1 / 2}\right\| \\
& \geq \frac{\beta \gamma_{2}}{2 \Delta t}\left(\left\|\delta_{x} \xi^{n+1}\right\|^{2}-\left\|\delta_{x} \xi^{n}\right\|^{2}\right) \\
& -\frac{\beta}{4}\left(2\left\|\delta_{x} \xi^{n+1}\right\|^{2}+2\left\|\delta_{x} \xi^{n}\right\|^{2}+\left\|\delta_{x} \widetilde{R}^{n+1 / 2}\right\|^{2}\right),
\end{aligned}
$$

$$
\begin{aligned}
I_{3_{2}} \geq & \frac{\beta \gamma_{2}}{2 \Delta t}\left(\left\|\delta_{y} \xi^{n+1}\right\|^{2}-\left\|\delta_{y} \xi^{n}\right\|^{2}\right) \\
& -\frac{\beta}{4}\left(2\left\|\delta_{y} \xi^{n+1}\right\|^{2}+2\left\|\delta_{y} \xi^{n}\right\|^{2}+\left\|\delta_{y} \widetilde{R}^{n+1 / 2}\right\|^{2}\right), \\
I_{4}= & \left(\frac{c^{2} \Delta t^{2}}{4} \delta_{x}^{2} \delta_{y}^{2} \frac{\eta^{n+1}-\eta^{n}}{\Delta t}, \frac{\eta^{n+1}+\eta^{n}}{2}\right) \\
= & \frac{1}{2 \Delta t} \frac{c \Delta t^{2}}{4}\left(\left\|\delta_{x} \delta_{y} \eta^{n+1}\right\|^{2}-\left\|\delta_{x} \delta_{y} \eta^{n}\right\|^{2}\right) \\
I_{5} & =\left(R^{n+1 / 2}, \frac{\eta^{n+1}+\eta^{n}}{2}\right) \\
& \leq \frac{1}{4}\left\|R^{n+1 / 2}\right\|^{2}+\frac{1}{2}\left(\left\|\eta^{n+1}\right\|^{2}+\left\|\eta^{n}\right\|^{2}\right) .
\end{aligned}
$$

Multiplying $2 \Delta t$ to both sides of the result derived from (29), we have

$$
\begin{aligned}
& \left(\left\|\eta^{n+1}\right\|^{2}-\left\|\eta^{n}\right\|^{2}\right) \\
& +2 \alpha \Delta t\left(\left\|\delta_{x}\left(\eta^{n+1}+\eta^{n}\right)\right\|^{2}+\left\|\delta_{y}\left(\eta^{n+1}+\eta^{n}\right)\right\|^{2}\right) \\
& +\beta \gamma_{2}\left(\left\|\delta_{x} \xi^{n+1}\right\|^{2}+\left\|\delta_{y} \xi^{n+1}\right\|^{2}-\left\|\delta_{x} \xi^{n}\right\|^{2}-\left\|\delta_{y} \xi^{n}\right\|^{2}\right) \\
& +\frac{c \Delta t^{2}}{4}\left(\left\|\delta_{x} \delta_{y} \eta^{n+1}\right\|^{2}-\left\|\delta_{x} \delta_{y} \eta^{n}\right\|^{2}\right)
\end{aligned}
$$




$$
\begin{aligned}
\leq & \frac{\Delta t}{2}\left(\beta\left\|\delta_{x} \widetilde{R}^{n+1 / 2}\right\|^{2}+\beta\left\|\delta_{y} \widetilde{R}^{n+1 / 2}\right\|^{2}+\left\|R^{n+1 / 2}\right\|^{2}\right) \\
& +\Delta t\left(\left\|\eta^{n+1}\right\|^{2}+\left\|\eta^{n}\right\|^{2}\right) \\
& +\beta\left(\left\|\delta_{x} \xi^{n+1}\right\|^{2}+\left\|\delta_{x} \xi^{n}\right\|^{2}+\left\|\delta_{y} \xi^{n+1}\right\|^{2}+\left\|\delta_{y} \xi^{n}\right\|^{2}\right) .
\end{aligned}
$$

Summing for $n$ to both sides of (30), we obtain

$$
\begin{aligned}
& \left\|\eta^{n}\right\|^{2}+\beta \gamma_{2}\left(\left\|\delta_{x} \xi^{n}\right\|^{2}+\left\|\delta_{y} \xi^{n}\right\|^{2}\right) \\
& \leq \frac{\Delta t}{2} \sum_{l=1}^{n-1}\left(\left\|R^{l+1 / 2}\right\|^{2}+\beta\left\|\delta_{x} \widetilde{R}^{l+1 / 2}\right\|^{2}+\beta\left\|\delta_{y} \widetilde{R}^{l+1 / 2}\right\|^{2}\right) \\
& +\Delta t \sum_{l=1}^{n-1}\left(2\left\|\eta^{l}\right\|^{2}+\beta\left\|\delta_{x} \xi^{l}\right\|^{2}+\beta\left\|\delta_{y} \xi^{l}\right\|^{2}\right)
\end{aligned}
$$

noticing that $\xi^{0}=\eta^{0}=0$, by Gronwall Lemma, we obtain the following theorem.

Theorem 2. Assume that $u, v$ are the accurate solutions of (6)-(9) with sufficient smoothness and $U, V$ are the difference solutions of (16)-(18). Let $\xi=u-U, \eta=v-V$, then there exists a positive constant $K$ independent of $\Delta t$ and $h$, such that

$$
\max _{n \leq[T / \Delta t]}\left(\left\|\eta^{n}\right\|+\left\|\delta_{x} \xi^{n}\right\|+\left\|\delta_{y} \xi^{n}\right\|\right) \leq K\left(\Delta t^{2}+h^{2}\right) .
$$

\section{Generalized Compact ADI Scheme}

In this section, we extend the method to compact ADI scheme for the numerical solution of the wave problems (1)(3).

4.1. Construction of the Compact ADI Scheme. Introducing

$$
\begin{array}{ll}
f_{x_{v}}=\frac{\partial^{2} v}{\partial x^{2}}, & f_{y_{v}}=\frac{\partial^{2} v}{\partial y^{2}}, \\
f_{x_{u}}=\frac{\partial^{2} u}{\partial x^{2}}, & f_{y_{u}}=\frac{\partial^{2} u}{\partial y^{2}} .
\end{array}
$$

We can rewrite (6) as follows:

$$
\frac{\partial v}{\partial t}-\alpha\left(f_{x_{v}}+f_{y_{v}}\right)-\beta\left(f_{x_{u}}+f_{y_{u}}\right)=S(x, y, t) .
$$

Using the fourth-order compact finite difference discretization $[6,8]$, we discretize (33) as follows:

$$
\begin{aligned}
& A_{x}\left(f_{x}\right)_{i, j}^{n}:=\frac{1}{12}\left(f_{x}\right)_{i-1, j}^{n}+\frac{5}{6}\left(f_{x}\right)_{i, j}^{n}+\frac{1}{12}\left(f_{x}\right)_{i+1, j}^{n}=\delta_{x}^{2} U_{i, j}^{n}, \\
& A_{y}\left(f_{y}\right)_{i, j}^{n}:=\frac{1}{12}\left(f_{y}\right)_{i, j-1}^{n}+\frac{5}{6}\left(f_{y}\right)_{i, j}^{n}+\frac{1}{12}\left(f_{y}\right)_{i, j+1}^{n}=\delta_{y}^{2} U_{i, j}^{n} .
\end{aligned}
$$

The Crank-Nicolson implicit discretization of (34) reads

$$
\begin{aligned}
& \frac{V_{i, j}^{n+1}-V_{i, j}^{n}}{\Delta t}-\frac{\alpha\left(\left(f_{x_{v}}\right)_{i, j}^{n+1}+\left(f_{x_{v}}\right)_{i, j}^{n}+\left(f_{y_{v}}\right)_{i, j}^{n+1}+\left(f_{y_{v}}\right)_{i, j}^{n}\right)}{2} \\
& \quad-\frac{\beta\left(\left(f_{x_{u}}\right)_{i, j}^{n+1}+\left(f_{x_{u}}\right)_{i, j}^{n}+\left(f_{y_{u}}\right)_{i, j}^{n+1}+\left(f_{y_{u}}\right)_{i, j}^{n}\right)}{2} \\
& =g_{i, j}^{n+1 / 2},
\end{aligned}
$$

where $i, j=1,2, \ldots, N-1$. Multiplying (36) by $\Delta t$, applying to its both sides with $A_{x} A_{y}$, and using the fact that the two operators $A_{x}$ and $A_{y}$ commute with each other, we obtain the implicit approximation of (6)-(9)

$$
\begin{gathered}
A_{x} A_{y}\left(V_{i, j}^{n+1}-V_{i, j}^{n}\right) \\
-\frac{\alpha \Delta t}{2}\left(A_{y} A_{x}\left(f_{x_{v}}\right)_{i, j}^{n+1}+A_{y} A_{x}\left(f_{x_{v}}\right)_{i, j}^{n}\right. \\
\left.+A_{x} A_{y}\left(f_{y_{v}}\right)_{i, j}^{n+1}+A_{x} A_{y}\left(f_{y_{v}}\right)_{i, j}^{n}\right) \\
-\frac{\beta \Delta t}{2}\left(A_{y} A_{x}\left(f_{x_{u}}\right)_{i, j}^{n+1}+A_{y} A_{x}\left(f_{x_{u}}\right)_{i, j}^{n}\right. \\
\left.+A_{x} A_{y}\left(f_{y_{u}}\right)_{i, j}^{n+1}+A_{x} A_{y}\left(f_{y_{u}}\right)_{i, j}^{n}\right) \\
=\Delta t A_{x} A_{y} S_{i, j}^{n+1 / 2}, \\
\frac{V_{i, j}^{n+1}+V_{i, j}^{n}}{2}=\gamma_{1} \frac{U_{i, j}^{n+1}+U_{i, j}^{n}}{2}+\gamma_{2} \frac{U_{i, j}^{n+1}-U_{i, j}^{n}}{\Delta t} .
\end{gathered}
$$

Let

$$
\begin{gathered}
\lambda_{1}=\frac{\Delta t}{\gamma_{1} \Delta t+2 \gamma_{2}}, \quad \lambda_{2}=\frac{\gamma_{1} \Delta t-2 \gamma_{2}}{\gamma_{1} \Delta t+2 \gamma_{2}}, \\
c=\alpha+\beta \lambda_{1}, \quad \gamma=\frac{1}{2} \beta\left(1+\lambda_{2}\right),
\end{gathered}
$$

then (37) can be conveniently written as

$$
\begin{aligned}
\left(A_{x} A_{y}\right. & \left.-\frac{c \Delta t}{2} A_{y} \delta_{x}^{2}-\frac{c \Delta t}{2} A_{x} \delta_{y}^{2}\right) V_{i, j}^{n+1} \\
= & \left(A_{x} A_{y}+\frac{c \Delta t}{2} A_{y} \delta_{x}^{2}+\frac{c \Delta t}{2} A_{x} \delta_{y}^{2}\right) V_{i, j}^{n} \\
& +\gamma \Delta t\left(A_{y} \delta_{x}^{2}+A_{x} \delta_{y}^{2}\right) U_{i, j}^{n}+\Delta t A_{x} A_{y} S_{i, j}^{n+1 / 2} .
\end{aligned}
$$


Adding perturbing term $\left(c^{2} \Delta t^{2} / 4\right) \delta_{x}^{2} \delta_{y}^{2}\left(V_{i, j}^{n+1}-V_{i, j}^{n}\right)$ to the left hand side of (40) and introducing the intermediate variable $V_{i, j}^{n+1 / 2}$, we obtain the compact PR ADI-like scheme as follows:

$$
\begin{aligned}
\left(A_{x}-\right. & \left.\frac{c \Delta t}{2} \delta_{x}^{2}\right) V_{i, j}^{n+1 / 2} \\
= & \left(A_{y}+\frac{c \Delta t}{2} \delta_{y}^{2}\right) V_{i, j}^{n}+\gamma\left(\frac{\Delta t}{2} \delta_{y}^{2}+\frac{1}{c} A_{y}\right) U_{i, j}^{n} \\
& +\frac{\Delta t}{2} A_{y} S_{i, j}^{n+1 / 2}, \\
\left(A_{y}-\frac{c \Delta t}{2} \delta_{y}^{2}\right) V_{i, j}^{n+1} & \left(A_{x}+\frac{c \Delta t}{2} \delta_{x}^{2}\right) V_{i, j}^{n+1 / 2}+\gamma\left(\frac{\Delta t}{2} \delta_{y}^{2}-\frac{1}{c} A_{y}\right) U_{i, j}^{n} \\
& +\frac{\Delta t}{2} A_{y} S_{i, j}^{n+1 / 2}, \\
& U_{i, j}^{n+1}=\lambda_{1}\left(V_{i, j}^{n+1}+V_{i, j}^{n}\right)-\lambda_{2} U_{i, j}^{n} .
\end{aligned}
$$

From (41) and (42), we obtain the boundary equation as follows:

$$
\begin{aligned}
A_{x} V_{i, j}^{n+1 / 2}= & A_{y} \frac{V_{i, j}^{n+1}+V_{i, j}^{n}}{2}+\frac{\gamma}{c} A_{y} U_{i, j}^{n} \\
& -\frac{c \Delta t}{4} \delta_{y}^{2}\left(V_{i, j}^{n+1}-V_{i, j}^{n}\right) .
\end{aligned}
$$

But usually we compute the boundary values of the intermediate using the following simple equality:

$$
V_{i, j}^{n+1 / 2}=\frac{V_{i, j}^{n+1}+V_{i, j}^{n}}{2}+\frac{\gamma}{c} U_{i, j}^{n}
$$

where $i=0, N, j=1, N-1$. In addition, following the idea of Douglas [2,3], we can get a compact Douglas scheme as follows:

$$
\begin{aligned}
\left(A_{x}-\frac{c \Delta t}{2} \delta_{x}^{2}\right)\left(V_{i, j}^{n+1 / 2}-V_{i, j}^{n}\right)= & c \Delta t\left(A_{y} \delta_{x}^{2}+A_{x} \delta_{y}^{2}\right) V_{i, j}^{n} \\
& +\gamma \Delta t\left(A_{y} \delta_{x}^{2}+A_{x} \delta_{y}^{2}\right) U_{i, j}^{n} \\
& +\Delta t A_{x} A_{y} g_{i, j}^{n+1 / 2} \\
\left(A_{y}-\frac{c \Delta t}{2} \delta_{y}^{2}\right)\left(V_{i, j}^{n+1}-V_{i, j}^{n}\right) & =V_{i, j}^{n+1 / 2}-V_{i, j}^{n} .
\end{aligned}
$$

The intermediate value of $V_{i, j}^{n+1 / 2}$ on the boundary is easy to be obtained from (47).
4.2. Error Estimate. We have derived a kind of compact PR ADI-like scheme in Section 4.1. In the following, we further analyze the error of the scheme. Expanding (41)-(42), we get

$$
\begin{gathered}
A_{x} A_{y} \frac{V_{i, j}^{n+1}-V_{i, j}^{n}}{\Delta t}-\alpha\left(A_{y} \delta_{x}^{2}+A_{x} \delta_{y}^{2}\right) \frac{V_{i, j}^{n+1}+V_{i, j}^{n}}{2} \\
-\beta\left(A_{y} \delta_{x}^{2}+A_{x} \delta_{y}^{2}\right) \frac{U_{i, j}^{n+1}+U_{i, j}^{n}}{2} \\
+\frac{c^{2} \Delta t^{2}}{4} \delta_{x}^{2} \delta_{y}^{2} \frac{V_{i, j}^{n+1}-V_{i, j}^{n}}{\Delta t}=A_{x} A_{y} S_{i, j}^{n+1 / 2} .
\end{gathered}
$$

Noting that $A_{x} U_{i, j}^{n}=\left(1+\left(h^{2} / 12\right) \delta_{x}^{2}\right) U_{i, j}^{n}, A_{y} U_{i, j}^{n}=(1+$ $\left.\left(h^{2} / 12\right) \delta_{y}^{2}\right) U_{i, j}^{n}$, (48) can be written as

$$
\begin{aligned}
\left(1+\frac{h^{2}}{12} \delta_{x}^{2}\right)\left(1+\frac{h^{2}}{12} \delta_{y}^{2}\right) \frac{V_{i, j}^{n+1}-V_{i, j}^{n}}{\Delta t} & -\alpha\left(\left(1+\frac{h^{2}}{12} \delta_{y}^{2}\right) \delta_{x}^{2}+\left(1+\frac{h^{2}}{12} \delta_{x}^{2}\right) \delta_{y}^{2}\right) \frac{V_{i, j}^{n+1}+V_{i, j}^{n}}{2} \\
& -\beta\left(\left(1+\frac{h^{2}}{12} \delta_{y}^{2}\right) \delta_{x}^{2}+\left(1+\frac{h^{2}}{12} \delta_{x}^{2}\right) \delta_{y}^{2}\right) \frac{U_{i, j}^{n+1}+U_{i, j}^{n}}{2} \\
& +\frac{c^{2} \Delta t^{2}}{4} \delta_{x}^{2} \delta_{y}^{2} \frac{V_{i, j}^{n+1}-V_{i, j}^{n}}{\Delta t} \\
= & \left(1+\frac{h^{2}}{12} \delta_{x}^{2}\right)\left(1+\frac{h^{2}}{12} \delta_{y}^{2}\right) S_{i, j}^{n+1 / 2} .
\end{aligned}
$$

Discretizing (6) as above and letting $\xi=u-U, \eta=v-V$, we obtain the error equations as follows:

$$
\begin{aligned}
& \left(1+\frac{h^{2}}{12} \delta_{x}^{2}\right)\left(1+\frac{h^{2}}{12} \delta_{y}^{2}\right) \frac{\eta_{i, j}^{n+1}-\eta_{i, j}^{n}}{\Delta t} \\
& -\alpha\left(\left(1+\frac{h^{2}}{12} \delta_{y}^{2}\right) \delta_{x}^{2}+\left(1+\frac{h^{2}}{12} \delta_{x}^{2}\right) \delta_{y}^{2}\right) \frac{\eta_{i, j}^{n+1}+\eta_{i, j}^{n}}{2} \\
& -\beta\left(\left(1+\frac{h^{2}}{12} \delta_{y}^{2}\right) \delta_{x}^{2}+\left(1+\frac{h^{2}}{12} \delta_{x}^{2}\right) \delta_{y}^{2}\right) \frac{\xi_{i, j}^{n+1}+\xi_{i, j}^{n}}{2} \\
& +\frac{c^{2} \Delta t^{2}}{4} \delta_{x}^{2} \delta_{y}^{2} \frac{\eta_{i, j}^{n+1}-\eta_{i, j}^{n}}{\Delta t}=R_{i, j}^{n+1 / 2},
\end{aligned}
$$

$$
\frac{\eta_{i, j}^{n+1}+\eta_{i, j}^{n}}{2}=\gamma_{1} \frac{\xi_{i, j}^{n+1}+\xi_{i, j}^{n}}{2}+\gamma_{2} \frac{\xi_{i, j}^{n+1}-\xi_{i, j}^{n}}{\Delta t}+\widetilde{R}_{i, j}^{n+1 / 2},
$$


where the truncation errors

$$
\begin{aligned}
R_{i, j}^{n+1 / 2}=\Delta t^{2}( & \frac{1}{24} A_{x} A_{y} \frac{\partial^{2} v}{\partial t^{3}}-\frac{\alpha}{8}\left(A_{y} \frac{\partial^{4} v}{\partial t^{2} \partial x^{2}}+A_{x} \frac{\partial^{4} v}{\partial t^{2} \partial y^{2}}\right) \\
& -\frac{\beta}{8}\left(A_{y} \frac{\partial^{4} u}{\partial t^{2} \partial x^{2}}+A_{x} \frac{\partial^{4} u}{\partial t^{2} \partial y^{2}}\right) \\
& +\frac{c^{2} \Delta t^{4}}{4} \frac{\partial^{5} v}{\partial t \partial x^{2} \partial y^{2}} \\
& -\frac{\alpha h^{4}}{240}\left(A_{y} \frac{\partial^{6} v}{\partial x^{6}}+A_{x} \frac{\partial^{6} v}{\partial y^{6}}\right) \\
& \left.-\frac{\beta h^{4}}{240}\left(A_{y} \frac{\partial^{6} u}{\partial x^{6}}+A_{x} \frac{\partial^{6} u}{\partial y^{6}}\right)\right)\left(x_{i}, y_{j}, t^{n+1 / 2}\right) \\
+ & O\left(\Delta t^{4}+\Delta t^{2} h^{4}+h^{6}\right), \\
\widetilde{R}_{i, j}^{n+1 / 2}= & \Delta t^{2}\left(\frac{1}{8} \frac{\partial^{2} v}{\partial t^{2}}-\frac{\gamma_{1}}{8} \frac{\partial^{2} u}{\partial t^{2}}-\frac{\gamma_{2}}{24} \frac{\partial^{3} u}{\partial t^{3}}\right)\left(x_{i}, y_{j}, t^{n+1 / 2}\right) \\
+ & O\left(\Delta t^{4}\right) .
\end{aligned}
$$

Hence there exist positive constants $C^{\prime}, C^{\prime \prime}$ such that

$$
\left|R_{i, j}^{n+1 / 2}\right| \leq C^{\prime}\left(\Delta t^{2}+h^{4}\right), \quad\left|\widetilde{R}_{i, j}^{n+1 / 2}\right| \leq C^{\prime \prime} \Delta t^{2} .
$$

Lemma 3 (see [1]). For $w \in V_{h}$, the following inequalities hold:

$$
\begin{array}{cl}
\frac{1}{4} h^{2}\left\|\delta_{x} w\right\|^{2} \leq\|w\|^{2}, & \frac{1}{4} h^{2}\left\|\delta_{y} w\right\|^{2} \leq\|w\|^{2}, \\
\frac{1}{4} h^{2}\left\|\delta_{x} \delta_{y} w\right\|^{2} \leq\left\|\delta_{x} w\right\|^{2}, & \frac{1}{4} h^{2}\left\|\delta_{x} \delta_{y} w\right\|^{2} \leq\left\|\delta_{y} w\right\|^{2} .
\end{array}
$$

From Lemmas 1 and 3 and Cauchy-Schwarz inequality, multiplying $\left(\left(\eta_{i, j}^{n+1}+\eta_{i, j}^{n}\right) / 2\right) h^{2}$ to both sides of $(50)$ and computing the inner product, we have

$$
\begin{aligned}
I_{1}= & \left(\left(1+\frac{h^{2}}{12} \delta_{x}^{2}\right)\left(1+\frac{h^{2}}{12} \delta_{y}^{2}\right) \delta_{t} \eta^{n}, \frac{\eta^{n+1}+\eta^{n}}{2}\right) \\
= & \left(\delta_{t} \eta^{n}, \frac{\eta^{n+1}+\eta^{n}}{2}\right)+\frac{h^{2}}{12}\left(\delta_{x}^{2} \delta_{t} \eta^{n}, \frac{\eta^{n+1}+\eta^{n}}{2}\right) \\
& +\frac{h^{2}}{12}\left(\delta_{y}^{2} \delta_{t} \eta^{n}, \frac{\eta^{n+1}+\eta^{n}}{2}\right) \\
& +\frac{h^{4}}{144}\left(\delta_{x}^{2} \delta_{y}^{2} \delta_{t} \eta^{n}, \frac{\eta^{n+1}+\eta^{n}}{2}\right) \\
= & \frac{1}{2 \Delta t}\left(\left\|\eta^{n+1}\right\|^{2}-\left\|\eta^{n}\right\|^{2}-\frac{h^{2}}{24 \Delta t}\left(\left\|\delta_{x} \eta^{n+1}\right\|^{2}-\left\|\delta_{x} \eta^{n}\right\|^{2}\right)\right. \\
& -\frac{h^{2}}{24 \Delta t}\left(\left\|\delta_{y} \eta^{n+1}\right\|^{2}-\left\|\delta_{y} \eta^{n}\right\|^{2}\right)
\end{aligned}
$$

$$
\begin{aligned}
& \left.+\frac{h^{4}}{288 \Delta t}\left(\left\|\delta_{x} \delta_{y} \eta^{n+1}\right\|^{2}-\left\|\delta_{x} \delta_{y} \eta^{n}\right\|^{2}\right)\right), \\
& I_{2_{1}}=-\left(\alpha\left(1+\frac{h^{2}}{12} \delta_{y}^{2}\right) \delta_{x}^{2} \frac{\eta^{n+1}+\eta^{n}}{2}, \frac{\eta^{n+1}+\eta^{n}}{2}\right) \\
& =\alpha\left\|\delta_{x} \frac{\eta^{n+1}+\eta^{n}}{2}\right\|^{2}-\frac{\alpha h^{2}}{12}\left\|\delta_{x} \delta_{y} \frac{\eta^{n+1}+\eta^{n}}{2}\right\|^{2} \\
& \geq \frac{\alpha h^{2}}{6}\left\|\delta_{x} \delta_{y} \frac{\eta^{n+1}+\eta^{n}}{2}\right\|^{2} \geq 0 \text {, } \\
& I_{2_{2}}=-\left(\alpha\left(1+\frac{h^{2}}{12} \delta_{x}^{2}\right) \delta_{y}^{2} \frac{\eta^{n+1}+\eta^{n}}{2}, \frac{\eta^{n+1}+\eta^{n}}{2}\right) \\
& =\alpha\left\|\delta_{y} \frac{\eta^{n+1}+\eta^{n}}{2}\right\|^{2}-\frac{\alpha h^{2}}{12}\left\|\delta_{x} \delta_{y} \frac{\eta^{n+1}+\eta^{n}}{2}\right\|^{2} \\
& \geq \frac{\alpha h^{2}}{6}\left\|\delta_{x} \delta_{y} \frac{\eta^{n+1}+\eta^{n}}{2}\right\|^{2} \geq 0 \text {, } \\
& I_{3_{1}}=-\left(\beta\left(1+\frac{h^{2}}{12} \delta_{y}^{2}\right) \delta_{x}^{2} \frac{\xi^{n+1}+\xi^{n}}{2}, \frac{\eta^{n+1}+\eta^{n}}{2}\right) \\
& =-\left(\beta \delta_{x}^{2} \frac{\xi^{n+1}+\xi^{n}}{2}, \frac{\eta^{n+1}+\eta^{n}}{2}\right) \\
& -\frac{\beta h^{2}}{12}\left(\delta_{x}^{2} \delta_{y}^{2} \frac{\xi^{n+1}+\xi^{n}}{2}, \frac{\eta^{n+1}+\eta^{n}}{2}\right) \\
& =\beta \gamma_{1}\left(\delta_{x} \frac{\xi^{n+1}+\xi^{n}}{2}, \delta_{x} \frac{\xi^{n+1}+\xi^{n}}{2}\right) \\
& +\beta \gamma_{2}\left(\delta_{x} \frac{\xi^{n+1}+\xi^{n}}{2}, \delta_{x} \frac{\xi^{n+1}-\xi^{n}}{\Delta t}\right) \\
& +\beta\left(\delta_{x} \frac{\xi^{n+1}+\xi^{n}}{2}, \delta_{x} \widetilde{R}^{n+1 / 2}\right) \\
& -\frac{\beta \gamma_{1} h^{2}}{12}\left(\delta_{x} \delta_{y} \frac{\xi^{n+1}+\xi^{n}}{2}, \delta_{x} \delta_{y} \frac{\xi^{n+1}+\xi^{n}}{2}\right) \\
& -\frac{\beta \gamma_{2} h^{2}}{12}\left(\delta_{x} \delta_{y} \frac{\xi^{n+1}+\xi^{n}}{2}, \delta_{x} \delta_{y} \frac{\xi^{n+1}-\xi^{n}}{\Delta t}\right) \\
& -\frac{\beta h^{2}}{12}\left(\delta_{x} \delta_{y} \frac{\xi^{n+1}+\xi^{n}}{2}, \delta_{x} \delta_{y} \widetilde{R}^{n+1 / 2}\right) \\
& \geq \frac{\beta \gamma_{2}}{2 \Delta t}\left(\left\|\delta_{x} \xi^{n+1}\right\|^{2}-\left\|\delta_{x} \xi^{n}\right\|^{2}\right) \\
& -\frac{h^{2}}{12} \frac{\beta \gamma_{2}}{2 \Delta t}\left(\left\|\delta_{x} \delta_{y} \xi^{n+1}\right\|^{2}-\left\|\delta_{x} \delta_{y} \xi^{n}\right\|^{2}\right) \\
& -\frac{\beta}{4}\left(\left\|\delta_{x} \xi^{n+1}\right\|^{2}+\left\|\delta_{x} \xi^{n}\right\|^{2}+\left\|\delta_{x} \widetilde{R}^{n+1 / 2}\right\|^{2}\right) \\
& -\frac{\beta h^{2}}{48}\left(\left\|\delta_{x} \delta_{y} \xi^{n+1}\right\|^{2}+\left\|\delta_{x} \delta_{y} \xi^{n}\right\|^{2}+\left\|\delta_{x} \delta_{y} \widetilde{R}^{n+1 / 2}\right\|^{2}\right)
\end{aligned}
$$




$$
\begin{aligned}
\geq & \frac{\beta \gamma_{2}}{2 \Delta t}\left(\left\|\delta_{x} \xi^{n+1}\right\|^{2}-\left\|\delta_{x} \xi^{n}\right\|^{2}\right) \\
& -\frac{h^{2}}{12} \frac{\beta \gamma_{2}}{2 \Delta t}\left(\left\|\delta_{x} \delta_{y} \xi^{n+1}\right\|^{2}-\left\|\delta_{x} \delta_{y} \xi^{n}\right\|^{2}\right) \\
& -\frac{\beta}{3}\left(2\left\|\delta_{x} \xi^{n+1}\right\|^{2}+2\left\|\delta_{x} \xi^{n}\right\|^{2}+\left\|\delta_{x} \widetilde{R}^{n+1 / 2}\right\|^{2}\right) \\
I_{3_{2}}= & -\left(\beta\left(1+\frac{h^{2}}{12} \delta_{x}^{2}\right) \delta_{y}^{2} \frac{\xi^{n+1}+\xi^{n}}{2}, \frac{\eta^{n+1}+\eta^{n}}{2}\right) \\
\geq & \frac{\beta \gamma_{2}}{2 \Delta t}\left(\left\|\delta_{y} e^{n+1}\right\|^{2}-\left\|\delta_{y} e^{n}\right\|^{2}\right) \\
& -\frac{h^{2}}{12} \frac{\beta \gamma_{2}}{2 \Delta t}\left(\left\|\delta_{x} \delta_{y} e^{n+1}\right\|^{2}-\left\|\delta_{x} \delta_{y} e^{n}\right\|^{2}\right) \\
& -\frac{\beta}{3}\left(2\left\|\delta_{y} \xi^{n+1}\right\|^{2}+2\left\|\delta_{y} \xi^{n}\right\|^{2}+\left\|\delta_{y} \widetilde{R}^{n+1 / 2}\right\|^{2}\right) \\
I_{5}= & \left(\| R^{n+1 / 2}, \frac{\eta^{n+1}+\eta^{n}}{2}\right) \\
I_{4}= & \frac{c^{2} \Delta t}{4}\left(\delta_{x}^{2} \delta_{y}^{2}\left(\eta^{n+1}-\eta^{n}\right), \frac{\eta^{n+1}+\eta^{n}}{2}\right) \\
= & \frac{c^{2} \Delta t}{8}\left(\left\|\delta_{x} \delta_{y} \eta^{n+1}\right\|^{2}-\left\|\delta_{x} \delta_{y} \eta^{n}\right\|^{2}\right) \\
& \left(\left\|R^{2}+2\right\| \eta^{n+1}\left\|^{2}+2\right\| \eta^{n} \|^{2}\right) \\
& -(\beta)
\end{aligned}
$$

Multiplying $2 \Delta t$ to both sides of the result derived from (55), we have

$$
\begin{gathered}
\left(\left\|\eta^{n+1}\right\|^{2}-\frac{h^{2}}{12}\left\|\delta_{x} \eta^{n+1}\right\|^{2}-\frac{h^{2}}{12}\left\|\delta_{y} \eta^{n+1}\right\|^{2}\right. \\
\left.+\frac{h^{4}}{144}\left\|\delta_{x} \delta_{y} \eta^{n+1}\right\|^{2}\right) \\
+\beta \gamma_{2}\left(\left\|\delta_{x} \xi^{n+1}\right\|^{2}-\frac{h^{2}}{12}\left\|\delta_{x} \delta_{y} \xi^{n+1}\right\|^{2}+\left\|\delta_{y} \xi^{n+1}\right\|^{2}\right. \\
\left.\quad-\frac{h^{2}}{12}\left\|\delta_{x} \delta_{y} \xi^{n+1}\right\|^{2}\right)+\frac{c^{2} \Delta t^{2}}{4}\left\|\delta_{x} \delta_{y} \eta^{n+1}\right\|^{2} \\
\leq\left(\left\|\eta^{n}\right\|^{2}-\frac{h^{2}}{12}\left\|\delta_{x} \eta^{n}\right\|^{2}-\frac{h^{2}}{12}\left\|\delta_{y} \eta^{n}\right\|^{2}+\frac{h^{4}}{144}\left\|\delta_{x} \delta_{y} \eta^{n}\right\|^{2}\right) \\
+\beta \gamma_{2}\left(\left\|\delta_{x} \xi^{n}\right\|^{2}-\frac{h^{2}}{12}\left\|\delta_{x} \delta_{y} \xi^{n}\right\|^{2}\right. \\
\left.+\left\|\delta_{y} \xi^{n}\right\|^{2}-\frac{h^{2}}{12}\left\|\delta_{x} \delta_{y} \xi^{n}\right\|^{2}\right)
\end{gathered}
$$

$$
\begin{aligned}
& +\frac{2 \beta \Delta t}{3}\left(2\left\|\delta_{x} \xi^{n+1}\right\|^{2}+2\left\|\delta_{x} \xi^{n}\right\|^{2}+\left\|\delta_{x} \widetilde{R}^{n+1 / 2}\right\|^{2}\right. \\
& \left.\quad+2\left\|\delta_{y} \xi^{n+1}\right\|^{2}+2\left\|\delta_{y} \xi^{n}\right\|^{2}+\left\|\delta_{y} \widetilde{R}^{n+1 / 2}\right\|^{2}\right) \\
& +\frac{c^{2} \Delta t^{2}}{4}\left\|\delta_{x} \delta_{y} \eta^{n}\right\|^{2} \\
& +\frac{\Delta t}{2}\left(\left\|R^{n+1 / 2}\right\|^{2}+2\left\|\eta^{n+1}\right\|^{2}+2\left\|\eta^{n}\right\|^{2}\right) .
\end{aligned}
$$

Summing for $n$ to both sides of (56), and by Lemma 3, we obtain

$$
\begin{gathered}
\frac{1}{3}\left\|\eta^{n}\right\|^{2}+\frac{2 \beta \gamma_{2}}{3}\left(\left\|\delta_{x} \xi^{n}\right\|^{2}+\left\|\delta_{y} \xi^{n}\right\|^{2}\right)+\frac{h^{4}}{144}\left\|\delta_{x} \delta_{y} \xi^{n}\right\|^{2} \\
\leq 2 \Delta t \sum_{l=1}^{n-1}\left\|\eta^{n}\right\|^{2}+\frac{8 \beta \Delta t}{3} \sum_{l=1}^{n-1}\left(\left\|\delta_{x} \xi^{n}\right\|^{2}+\left\|\delta_{y} \xi^{n}\right\|^{2}\right) \\
+\frac{\Delta t}{2} \sum_{l=1}^{n-1}\left\|R^{n+1 / 2}\right\|^{2} \\
+\frac{2 \beta \Delta t}{3} \sum_{l=1}^{n-1}\left(\left\|\delta_{x} \widetilde{R}^{n+1 / 2}\right\|^{2}+\left\|\delta_{y} \widetilde{R}^{n+1 / 2}\right\|^{2}\right) .
\end{gathered}
$$

Noticing that $\xi^{0}=\eta^{0}=0$, by Gronwall Lemma, we obtain the following theorem.

Theorem 4. Assume that $u, v$ are the accurate solutions of (6)-(9) with sufficient smoothness and $U, V$ are the difference solutions of (41)-(43). Let $\xi=u-U, \eta=v-V$, then there exists a positive constant $K^{\prime}$ independent of $\Delta t$ and $h$, such that

$$
\begin{aligned}
& \max _{n \leq[T / \Delta t]}\left(\left\|\eta^{n}\right\|+\left\|\delta_{x} \xi^{n}\right\|+\left\|\delta_{y} \xi^{n}\right\|+\frac{h^{4}}{144}\left\|\delta_{x} \delta_{y} \xi^{n}\right\|\right) \\
& \quad \leq K^{\prime}\left(\Delta t^{2}+h^{4}\right) .
\end{aligned}
$$

\section{Numerical Experiment}

In this section, we exemplify two numerical examples to illustrate the effectiveness of the present ADI scheme. Lele [9] discussed some finite differencing errors for first and second derivatives by establishing the relationship of wave number (for differential equation) and numerical wave number (for difference scheme) and indicated that standard Pade scheme (which is known as compact finite difference scheme in this paper) is a good choice to discrete differential equations. In this paper, we focus on the efficient alternating direction technique for solving second-order wave equations. Because the compact method in spatial direction is standard, the scheme is effective.

The computer language used for the programming is Fortran, and the programs are performed on professional computer (CPU is $3.10 \mathrm{GHz}$, and Memory is $3.47 \mathrm{G}$ ). For convenience, we denote the present second-order ADI scheme (16)-(18) by PR, the present compact ADI scheme (41)-(43) 
TABLE 1: The $L^{2}$-norm and CPU time for the second-order schemes.

\begin{tabular}{lcccc}
\hline \multirow{2}{*}{ Scheme } & \multicolumn{2}{c}{$N=40, \Delta t=h$} & \multicolumn{2}{c}{$N=60, \Delta t=h$} \\
& $L^{2}$-norm & $\begin{array}{c}\text { CPU time } \\
(\mathrm{s})\end{array}$ & $L^{2}$-norm & $\begin{array}{c}\text { CPU time } \\
(\mathrm{s})\end{array}$ \\
\hline PR & $5.8154 \times 10^{-4}$ & 0.125000 & $2.5959 \times 10^{-4}$ & 0.406250 \\
Dou & $5.7463 \times 10^{-4}$ & 0.125000 & $2.5751 \times 10^{-4}$ & 0.390625 \\
LOD & $6.2814 \times 10^{-3}$ & 0.140625 & $3.9884 \times 10^{-3}$ & 0.437500 \\
$(\theta=0.25)$ & & & & \\
LOD & $6.3956 \times 10^{-3}$ & 0.140625 & $4.0402 \times 10^{-3}$ & 0.437500 \\
$(\theta=0.5)$ & & & & \\
\hline
\end{tabular}

TABLE 2: The $L^{2}$-norm and CPU time for the fourth-order schemes.

\begin{tabular}{lcccc}
\hline \multirow{2}{*}{$\begin{array}{c}\text { Scheme } \\
\end{array}$} & $L^{2}$-norm & $\begin{array}{c}\text { CPU time } \\
(\mathrm{s})\end{array}$ & $L^{2}$-norm & $\begin{array}{c}\text { CPU time } \\
(\mathrm{s})\end{array}$ \\
\hline CPR & $5.6026 \times 10^{-7}$ & 0.375000 & $3.5894 \times 10^{-7}$ & 5.156250 \\
CDou & $5.1636 \times 10^{-7}$ & 0.562500 & $3.3038 \times 10^{-7}$ & 6.578125 \\
LOD & $5.1383 \times 10^{-4}$ & 0.390625 & $1.2695 \times 10^{-4}$ & 5.265625 \\
$(\theta=0.25)$ & & & & \\
LOD & $5.1397 \times 10^{-4}$ & 0.390625 & $1.2694 \times 10^{-4}$ & 5.265625 \\
$(\theta=0.5)$ & & & & \\
\hline
\end{tabular}

by CPR, the second-order ADI scheme (21)-(22) by Dou, the compact ADI scheme (46)-(47) by CDou, and the three-level LOD scheme by LOD.

Example 5. Let $\gamma_{1}=1.0, \gamma_{2}=1.0, a=1.0, q=1.0$, and $S=\left(\pi^{2}-3 \pi+2\right) \mathrm{e}^{-\pi t} \sin (x+y)$ in (1), then the true solution is $u=\mathrm{e}^{-\pi t} \sin (x+y)$. We chose different grids and compared the accuracy of the computed solutions with other schemes. The solutions were obtained for $t_{\max }=2.0$. The quantities that we compared are the maximum $L^{2}$-norm and the total elapsed time (CPU) in seconds.

Tables 1 and 2 display the $L^{2}$-norm errors and the CPU time. From Table 1, one can easily see that the error from the present second-order scheme is smaller than that from other schemes, and it converges as fast as other schemes. From Table 2, we can find the superiority of the present compact ADI scheme, which has the highest accuracy and fastest computational efficiency. In Figures 1 and 2, we plot the errors and cost time at various grids. The figures show the superiority of the compact ADI over other schemes (secondorder scheme). The CPR has much higher and more efficiency than CDou, which may be the best scheme to solve above questions.

Example 6. Let $\gamma_{1}=1.0, \gamma_{2}=1.0, a=1.0, q=1.0$, and $S=\pi^{2} \sin 5 \pi x \sin 2 \pi y\left[34+\left(0.5+17 \pi^{2}\right) \cos \pi^{2} t+\right.$ $\left.\left(17-0.5 \pi^{2}\right) \sin \pi^{2} t\right]$ in (1). The exact solution is $u=(1+$ $\left.0.5 \sin \pi^{2} t\right) \sin 5 \pi x \cos 3 \pi y$, and the initial and boundary conditions can be obtained based on the exact solution. We chose the grids $25 \times 25$ and $50 \times 50$, and the time step with $\Delta t=h^{2}$. The solutions were obtained for $t_{\max }=5.0$. We compared the accuracy of the computed solutions from



FIGURE 1: The error of all schemes for various grid sizes when $\Delta t=$ $h^{2}$.



FIGURE 2: The CPU time of all schemes for various grid sizes when $\Delta t=h^{2}$.

the present ADI scheme and other four ADI schemes. The quantities that we compared are the $L^{2}$-norm and the total elapsed time (CPU) in seconds.

Table 3 lists $t$ the total elapsed time (CPU) in seconds and the $L^{2}$-norm error are listed in Table 3 and shown in Figures 3 and 4 . One can see that the error from the same order scheme is very close. From the table and figures, we can see that the present fourth-order scheme not only has high accuracy but also converges as fast as other schemes, especially for CPR scheme.

From Figures 1 and 3, it is easily to find that the tested methods degrade to first-order method as the grid 
TABLE 3: The $L^{2}$-norm and CPU time for all schemes.

\begin{tabular}{lcccc}
\hline \multirow{2}{*}{ Scheme } & \multicolumn{2}{c}{$N=25, \Delta t=h^{2}$} & \multicolumn{2}{c}{$N=50, \Delta t=h^{2}$} \\
& $L^{2}$-norm & CPU time (s) & $L^{2}$-norm & CPU time (s) \\
\hline PR & $3.9262 \times 10^{-2}$ & 1.8125 & $1.0218 \times 10^{-2}$ & 23.7812 \\
CPR & $8.0830 \times 10^{-4}$ & 1.9375 & $5.2720 \times 10^{-5}$ & 24.8906 \\
Dou & $3.9262 \times 10^{-2}$ & 1.8281 & $1.0218 \times 10^{-2}$ & 23.0625 \\
CDou & $8.0830 \times 10^{-4}$ & 2.0156 & $5.2720 \times 10^{-5}$ & 29.0312 \\
LOD & $4.2503 \times 10^{-1}$ & 2.0508 & $6.5634 \times 10^{-2}$ & 25.6705 \\
\hline
\end{tabular}

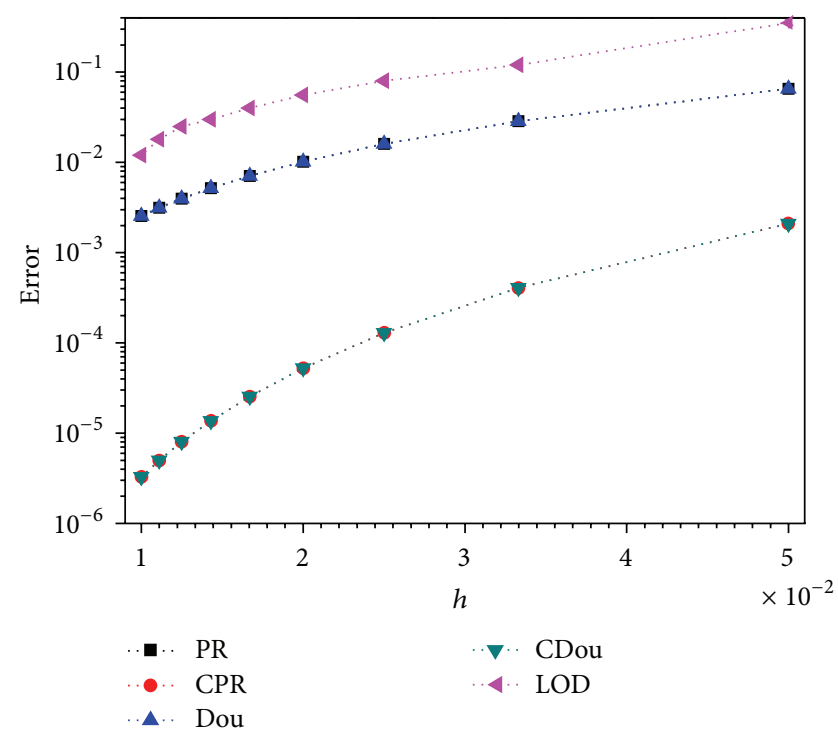

FIGURE 3: The error of all schemes for various grid sizes when $\Delta t=$ $h^{2}$.

gets coarse. In all schemes, we add perturbing term $\left(c^{2} \Delta t / 4\right) \delta_{x}^{2} \delta_{y}^{2}\left(V_{i, j}^{n+1}-V_{i, j}^{n}\right)$ that is, an approximation to $\left(c^{2} \Delta t^{2} / 4\right)\left(\partial 5 v / \partial t \partial x^{2} \partial y^{2}\right)$. When step $h$ becomes large, the time step $\Delta t$ varies correspondingly because of $\Delta t=h$ for the second-order schemes or $\Delta t=h^{2}$ for the compact schemes. Hence, the effect of perturbing term is enhanced, which results in the schemes seem to degrade to first-order methods as the grid gets coarse. We can choose suitable $h$ and get required accuracy.

\section{Conclusions}

In this paper, we proposed two new ADI schemes for solving nonhomogeneous wave problems. It has been proved that they are unconditionally stable. The compact scheme proposed in this paper is fourth-order accurate in space and second-order accurate in time and allows a considerable saving in computing time. Numerical examples are given to test their high accuracy and to show their superiority over some other schemes in terms of accuracy and computational costs.

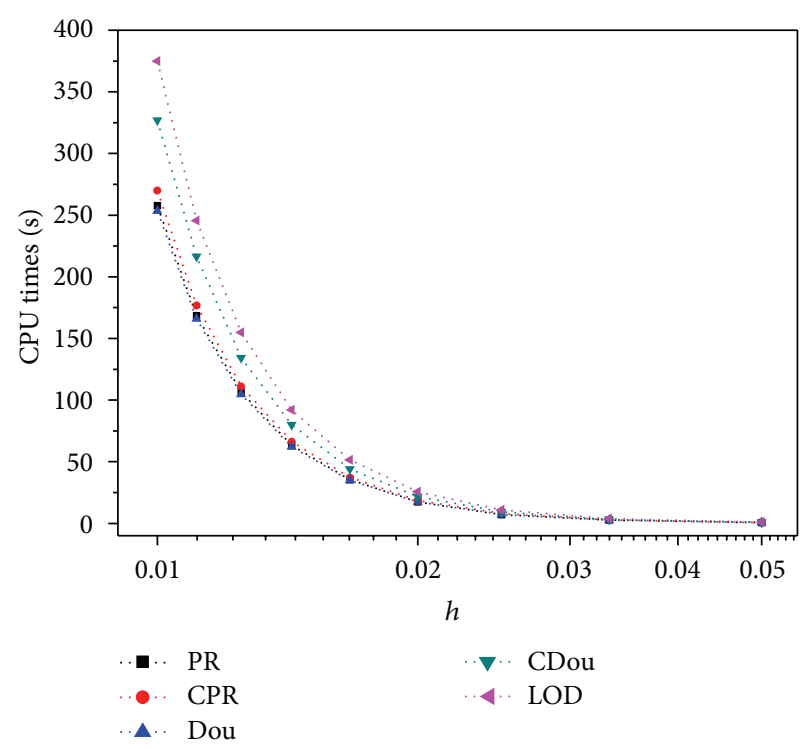

FIGURE 4: The CPU time of all schemes for various grid sizes when $\Delta t=h^{2}$.

The present scheme can be applied to the following variable coefficient differential models on domain $\Omega=$ $[0,1]^{2}$ :

$$
\begin{array}{r}
\gamma_{1} \frac{\partial u}{\partial t^{2}}+\gamma_{2} \frac{\partial^{2} u}{\partial t^{2}}-\nabla \cdot(A \nabla u)-\frac{\partial}{\partial t} \nabla \cdot(Q \nabla u)=S, \\
(x, y) \in \Omega, t \in(0, T], \\
u(x, y, 0)=u_{0}(x, y), \quad u_{t}(x, y, 0)=\psi(x, y), \\
(x, y) \in \Omega, \\
u(x, y, t)=\varphi(x, y, t), \quad(x, y) \in \partial \Omega, t \in(0, T],
\end{array}
$$

where $A=A(x, y)$ and $Q=Q(x, y)$ are diagonal, nonnegative diffusion tensors.

\section{Acknowledgment}

This work is partly supported by National Magnetic Confinement Fusion Science Program, Grant no. 2011GB112004.

\section{References}

[1] A. J. Cheng, "Improvement of stability and convergence for Douglas scheme in two space variables," Numerical Mathematics: A Journal of Chinese Universities, vol. 20, no. 3, pp. 265-272, 1998.

[2] S. Kim and H. Lim, "High-order schemes for acoustic waveform simulation," Applied Numerical Mathematics, vol. 57, no. 4, pp. 402-414, 2007.

[3] Z. Zhang and D. Deng, "A new alternating-direction finite element method for hyperbolic equation," Numerical Methods for Partial Differential Equations, vol. 23, no. 6, pp. 1530-1559, 2007. 
[4] C. Johnson, Numerical Solutions of Partial Differential Equations by the Finite Element Method, Cambridge University Press, New York, NY, USA, 1987.

[5] J. Douglas Jr. and J. E. Gunn, "A general formulation of alternating direction methods-part I: parabolic and hyperbolic problems," Numerische Mathematik, vol. 6, no. 1, pp. 428-453, 1964.

[6] J. Douglas Jr. and S. Kim, "Improved accuracy for locally onedimensional methods for parabolic equations," Mathematical Models and Methods in Applied Sciences, vol. 11, no. 9, pp. 15631579, 2001.

[7] J. Douglas Jr. and D. W. Peaceman, "Numerical solution for two-dimensional heat flow problems," American Institute of Chemical Engineers Journal, vol. 1, pp. 505-512, 1955.

[8] S. Karaa, "A high-order compact ADI method for solving three-dimensional unsteady convection-diffusion problems," Numerical Methods for Partial Differential Equations, vol. 22, no. 4, pp. 983-993, 2006.

[9] S. K. Lele, "Compact finite difference schemes with spectral-like resolution," Journal of Computational Physics, vol. 103, no. 1, pp. 16-42, 1992.

[10] J. Li, Y. Chen, and G. Liu, "High-order compact ADI methods for parabolic equations," Computers and Mathematics with Applications, vol. 52, no. 8-9, pp. 1343-1356, 2006.

[11] H. Lim, S. Kim, and J. Douglas Jr., "Numerical methods for viscous and nonviscous wave equations," Applied Numerical Mathematics, vol. 57, no. 2, pp. 194-212, 2007.

[12] M. Li, T. Tang, and B. Fornberg, "A compact fourth-order finite difference scheme for the steady incompressible NavierStokes equations," International Journal for Numerical Methods in Fluids, vol. 20, no. 10, pp. 1137-1151, 1995.

[13] D. W. Peaceman and H. Rachford, "The numerical solution of parabolic and elliptic differential equations," Journal of the Society for Industrial and Applied Mathematics, vol. 3, pp. 2841, 1959.

[14] J. Zhang, "Multigrid method and fourth-order compact scheme for 2D Poisson equation with unequal mesh-size discretization," Journal of Computational Physics, vol. 179, no. 1, pp. 170-179, 2002.

[15] D. W. Peaceman and H. H. Rachford Jr., "The numerical solution of parabolic and elliptic differential equations," Journal of the Society for Industrial and Applied Mathematics, vol. 3, pp. 28-42, 1955. 


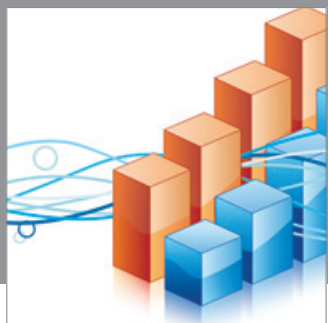

Advances in

Operations Research

mansans

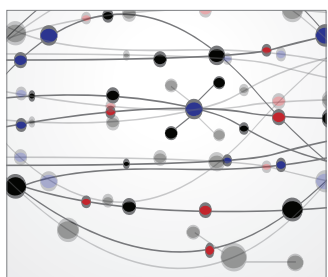

The Scientific World Journal
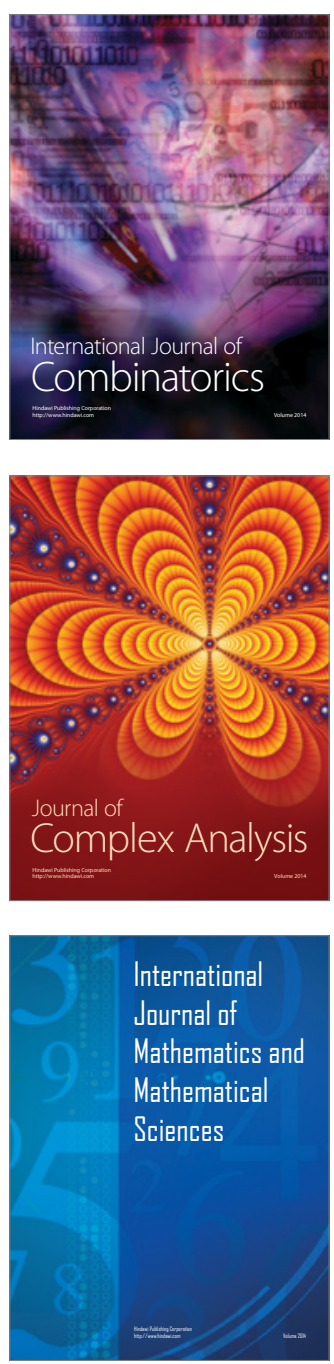
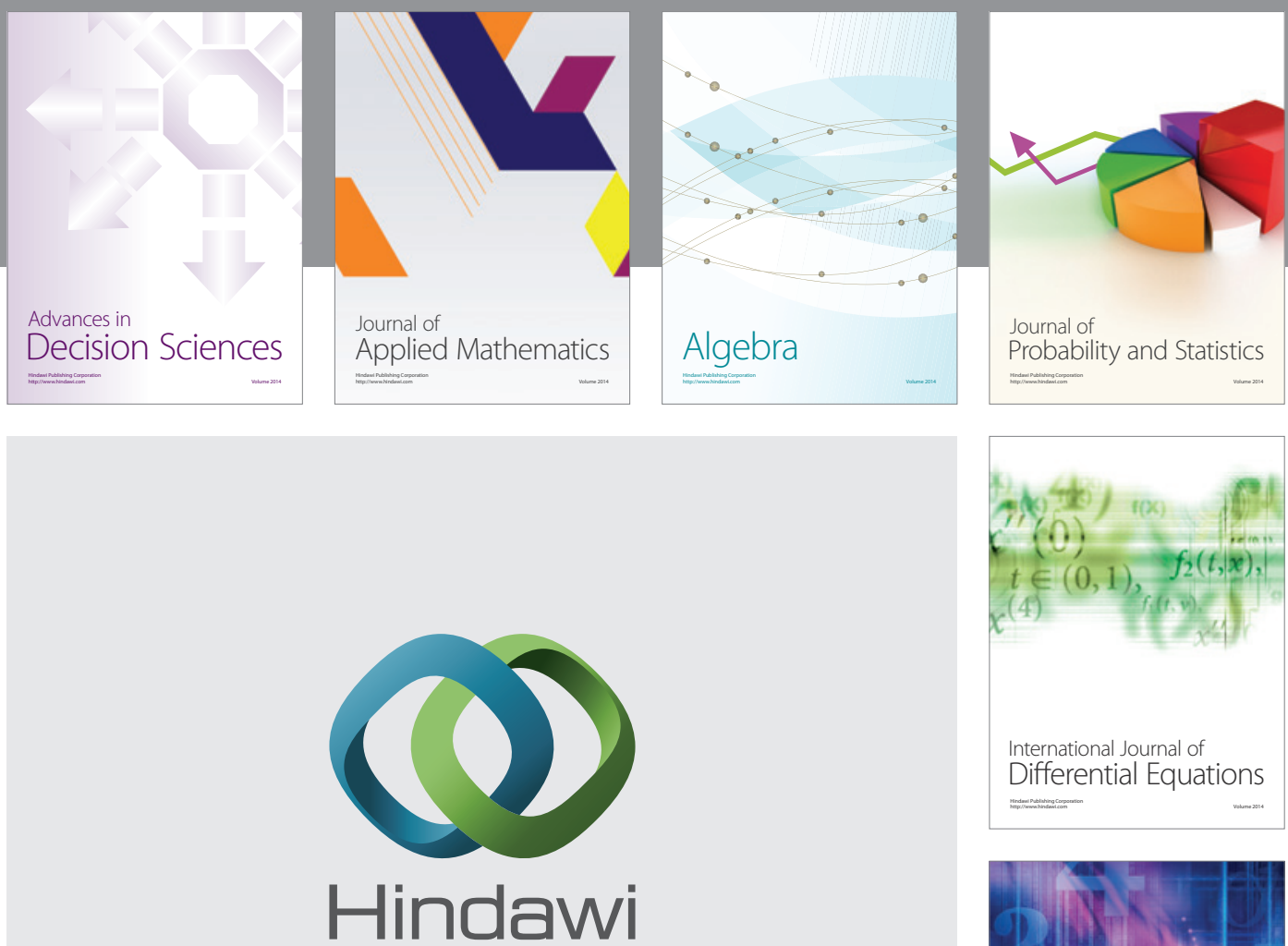

Submit your manuscripts at http://www.hindawi.com
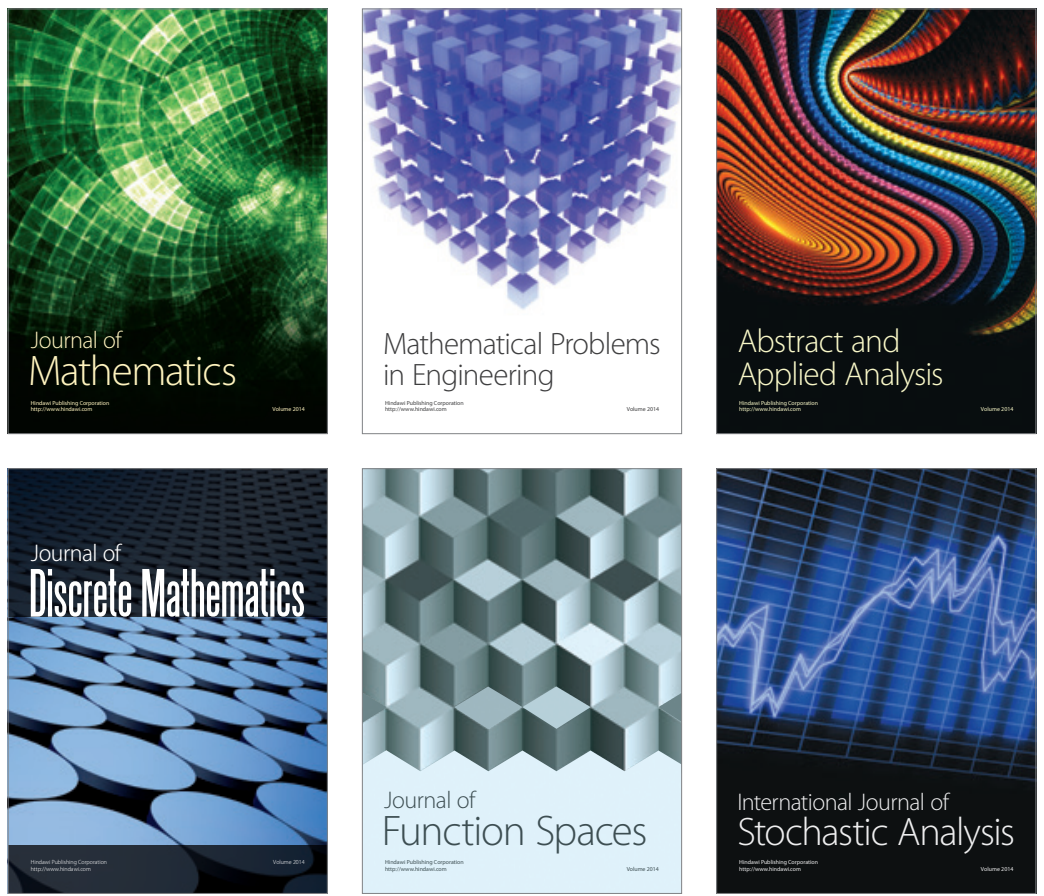

Journal of

Function Spaces

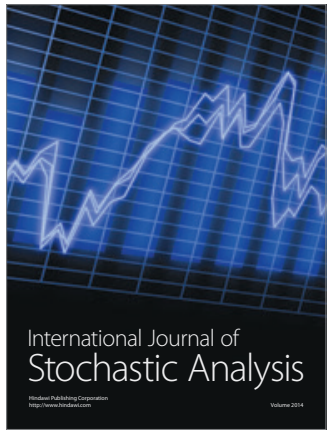


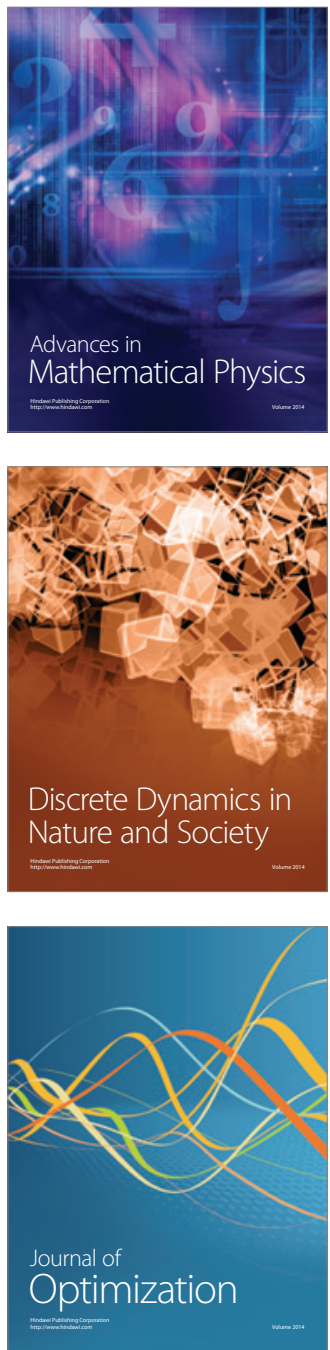